\title{
Histone deacetylase HDAC4 promotes the proliferation and invasion of glioma cells
}

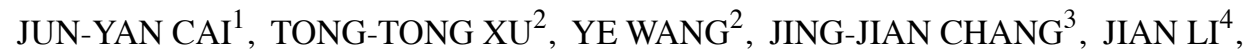 \\ XIAO-YANG CHEN ${ }^{3}, \mathrm{XI} \mathrm{CHEN}^{3}$, YI-FEI YIN ${ }^{3}$ and XUE-JUN NI ${ }^{3}$ \\ ${ }^{1}$ Department of Rehabilitation, Affiliated Hospital of Nantong University, Nantong, Jiangsu 226001; \\ ${ }^{2}$ School of Medicine, Nantong University, Nantong, Jiangsu 226001; Departments of ${ }^{3}$ Ultrasound and \\ ${ }^{4}$ Neurosurgery, Affiliated Hospital of Nantong University, Nantong, Jiangsu 226001, P.R. China
}

Received January 11, 2018; Accepted August 7, 2018

DOI: 10.3892/ijo.2018.4564

\begin{abstract}
Glioma is the most lethal type of primary brain tumor characterized by aggressiveness and a poor prognosis. Histone deacetylase 4 (HDAC4) is frequently dysregulated in human malignancies. However, its biological functions in the development of glioma are not fully understood. The present study aimed to evaluate HDAC4 expression in human glioma and to elucidate the mechanistic role of HDAC4 in glioma. The results suggested that HDAC4 was significantly upregulated in glioma tissues and a number of glioma cell lines compared with adjacent non-tumor tissues and the non-cancerous human glial cell line SVG p12, respectively $(\mathrm{P}<0.05)$. The proliferation, adenosine triphosphate (ATP) levels and invasion ability were substantially enhanced in U251 cells with HDAC4 overexpression, and suppressed in U251 cells with a knockdown of HDAC4 compared with that in U251 cells transfected with the negative control. Knockdown of HDAC4 resulted in cell cycle arrest at the G0/G1 phase and induced the increase of reactive oxygen species level in U251 cells. Furthermore, HDAC4 overexpression was revealed to substantially inhibit the expression of cyclin-dependent kinase (CDK) inhibitors p21 and p27, and the expression of E-cadherin and $\beta$-catenin in glioma U251 cells. Knockdown of HDAC4 substantially promoted the expression of CDK1 and CDK2 and vimentin in glioma U251 cells. Mechanistically, the results of the present study demonstrated that HDAC4 displayed a significant upregulation in glioma, and promoted glioma cell proliferation and invasion mediated through the repression of p21, p27, E-cadherin and $\beta$-catenin, and the potentiation of CDK1, CDK2 and vimentin. Altogether, the present study revealed that HDAC4 overexpression was central for the tumorigenesis
\end{abstract}

Correspondence to: Professor Xue-Jun Ni, Department of Ultrasound, Affiliated Hospital of Nantong University, 20 Xishi Road, Nantong, Jiangsu 226001, P.R. China

E-mail: ultrasound_ni@163.com

Key words: glioma, histone deacetylase 4, proliferation, invasion of glioma, which may serve as a useful prognostic biomarker and potential therapeutic target for glioma.

\section{Introduction}

Glioma is the most lethal type of primary malignant brain tumor characterized by extreme proliferation and aggressive invasion $(1,2)$. The invasiveness of glioma cells is the most difficult obstacle to combat in order to improve prognosis. Diffuse infiltrative gliomas account for $\sim 80 \%$ of all malignant brain tumor types, which results in a poor prognosis despite the advancements made in developing therapeutic strategies for malignant glioma $(3,4)$. Furthermore, heterogeneity is the foremost feature of glioma, which makes the diagnosis and selection of appropriate treatments more difficult. Therefore, the identification of genes associated with glioma invasiveness and heterogeneity has become a research focus. Over the last decade, substantial progress has been made in elucidating the underlying genetic causes of glioma (5). The expression of RAB34, member RAS oncogene family confers a poor prognosis in patients with high-grade glioma (6). Suppressor of cytokine signaling 3 promoter methylation promotes glioma cell invasion through signal transducer and activator of transcription 3 and focal adhesion kinase 1 activation $(7,8)$. However, the role of histone deacetylase 4 (HDAC4) in glioma cells has never been investigated.

HDAC4, a member of the class IIa family of HDACs, is known to undergo dynamic shuttling between the nucleus and cytoplasm and to function as a transcriptional corepressor that has been associated with a number of cellular and epigenetic processes (9). Cohen et al (10) revealed that the differential phosphorylation and localization of HDAC4 was conducive to setting up fiber type-specific transcriptional programs. Mitogen-activated protein kinase $\mathrm{p} 38$ promoted the degradation of HDAC4, which released Runt related transcription factor 2 and contributed to chondrocyte hypertrophy and bone formation (11). Accumulating evidence indicates that HDAC4 serves a crucial role in tumorigenesis and is frequently dysregulated in human malignancies (12). HDAC4 served a central role in breast cancer growth and invasion. HDAC4 knockdown by RNA interference inhibited breast cancer cell growth, migration and invasion through downregulating Ki-67 
and matrix metalloproteinase-2 (MMP-2) (13). The nuclear import of HDAC4 is required for melatonin-induced apoptosis in colorectal cancer LoVo cells (14). HDAC4 displayed a significant upregulation in multiple myeloma, and the downregulation of HDAC4 suppressed survival and migration and promoted apoptosis and autophagy of multiple myeloma cells compared with multiple myeloma cells with HDAC4 overexpression $(\mathrm{P}<0.05)$, respectively (15). HDAC4 may bind with RELB proto-oncogene, nuclear factor- $\kappa \beta$ subunit (RelB)-p52 forming an HDAC4-RelB-p52 complex, which maintains repressive chromatin around $\mathrm{Bcl} 2$ modifying factor and $\mathrm{Bcl} 2$ interacting mediator of cell death and regulates the survival and growth of multiple myeloma cells. Disruption of the HDAC4-RelB-p52 complex by a HDAC4-mimetic polypeptide blocks the growth of multiple myeloma (16). Zeng et al (17) demonstrated that HDAC4 was overexpressed in esophageal squamous cell carcinoma, and HDAC4 overexpression was associated with an advanced clinical stage and poor survival. HDAC4 inhibition sensitized lung cancer A549 cells to doxorubicin resistance by reducing the phosphorylation of signal transducers and activators of transcription-1 (STAT1) and the expression of epidermal growth factor receptor (EGFR), which suggested an interaction between HDAC4, STAT1 and EGFR (18). In summary, HDAC4 has been revealed to serve an important role in tumorigenesis, metastasis and invasion. However, little is known regarding the specific function of HDAC4 in glioma.

In the present study, glioma cell lines (U251, U-87MG and LN-18) and the non-cancerous human glial cell line SVG p12 cells were used to detect the expression of HDAC4 by western blotting. The effects of HDAC4 on U251 cell proliferation, adenosine triphosphate (ATP) and reactive oxygen species (ROS) generation, the cell cycle and cell invasion were analyzed. In addition, the effects of HDAC4 on the expression of cell cycle-associated proteins p21, p27 and CDK1 were analyzed, and epithelial-mesenchymal transition (EMT)associated proteins vimentin, E-cadherin and $\beta$-catenin in U251 cells. These data may be useful in the prediction of glioma prognosis and the establishment of targeted therapies.

\section{Materials and methods}

Specimens. A total of 12 paired glioma tissues and adjacent non-tumor tissues were collected from patients $(7$ male and 5 female; aged 12-65 years, mean age, 39.4 \pm 16.1 years and $30.5 \pm 15.2$ years, respectively) with glioma that underwent routine resection surgery at the Department of Neurosurgery of the Affiliated Hospital of Nantong University (Nantong, China) between January 2014 and November 2016. All patients had not received any other treatment prior to surgery, including radiotherapy and chemotherapy. The gliomas included 1 grade I, 3 grade II, 5 grade III and 3 grade IV [2007 World Health Organization classification of tumors of the central nervous system (19)]. All tissue specimens were obtained with written informed consent and ethically approved by the Human Ethics Committee of The Affiliated Hospital of Nantong University. All tissue samples were immediately frozen in liquid nitrogen and stored at $-80^{\circ} \mathrm{C}$ for further experiments.

Materials. All cell culture reagents, including fetal bovine serum, minimum essential medium (MEM), sodium pyru- vate, L-glutamine, penicillin, streptomycin and HEPES, were purchased from Gibco (Thermo Fisher Scientific, Inc., Waltham, MA, USA). Human glioblastoma cell lines U251 and LN-18, U-87MG (from glioblastoma of unknown origin which was authenticated by STR profiling) and non-cancerous human glial cell line SVG p12 cells [identified as being infected with BK polyomavirus strain UT (20). There was no bearing on the results obtained using SVG p12 cells] were purchased from the American Type Culture Collection (Manassas, VA, USA). Radio immunoprecipitation assay (RIPA) lysis buffer, bicinchoninic acid (BCA) assay kit, Beyo electrochemiluminesence (ECL) moon, polyvinylidene difluoride (PVDF) membrane, ROS assay kit, cell counting kit-8, enhanced ATP assay kit and propidium iodide (PI) were obtained from the Beyotime Institute of Biotechnology (Haimen, China). Transwell invasion filter was supplied by Costar (Corning Incorporated, Corning, NY, USA). Matrigel was purchased from Collaborative Biomedical Products (Bedford, MA, USA). The antibodies used in the present study include: Rabbit anti-HDAC4 polyclonal antibody (cat. no. 2072S; Cell Signaling Technology, Inc., Danvers, MA, USA), rabbit anti-p21 cyclin dependent kinase (CDK) inhibitor 1A/WAF1, anti-p27 ${ }^{\mathrm{Kip} 1 / \mathrm{CDK} \text { inhibitor 1B }}$ and anti-CDK1/cell division cycle 2 polyclonal antibodies (cat. nos. LS-C136937-100, LS-C17115-100 and LS-C402186-60, respectively; LifeSpan BioSciences, Inc., Seattle, WA, USA), rabbit anti-vimentin and anti- $\beta$-catenin polyclonal antibodies (cat. nos. PB9359 and PA1212, respectively; Boster Biological Technology, Pleasanton, CA, USA), rabbit anti-E-cadherin antibody (cat.no. ab155833; Abcam, Cambridge, UK), rabbit anti-GAPDH antibody (cat. no. orb69587; Biorbyt Ltd., Cambridge, UK), horseradish peroxidase-conjugated goat anti-rabbit immunoglobulin $\mathrm{G}$ secondary antibody polyclonal antibody (cat. no. NBP2-30348H; Novus Biologicals, LLC, Littleton, CO, USA).

Cell culture and treatment. Human glioma cell lines (U251, U-87MG and LN-18) and non-cancerous human fetal glial (SVG p12) cells were all cultured in MEM complemented with $10 \%$ fetal bovine serum (FBS), $1 \mathrm{mM}$ sodium pyruvate, $2 \mathrm{mM}$ L-glutamine, $50 \mathrm{U} / \mathrm{ml}$ penicillin, $50 \mu \mathrm{g} / \mathrm{ml}$ streptomycin and $10 \mathrm{mM}$ HEPES at $37^{\circ} \mathrm{C}$ in a humidified atmosphere with $5 \% \mathrm{CO}_{2}$.

$\mathrm{U} 251$ cells were selected to perform further studies. A total of $2 \times 10^{5} \mathrm{U} 251$ cells were cultured in a 6-well plate with $2 \mathrm{ml}$ antibiotic-free MEM medium containing 10\% FBS. U251 cells, grown at $80 \%$ confluence, were transfected with HDAC4 small interfering RNA (siRNA; $100 \mathrm{nM}$; cat. no. stB0001595C-1-5; Guangzhou RiboBio Co., Ltd., Guangzhou, China) or pcDNA3.1-HDAC4 (Shanghai GeneChem Co., Ltd, Shanghai, China) for $6 \mathrm{~h}$ at $37^{\circ} \mathrm{C}$ using Lipofectamine ${ }^{\circledR} 2000$ (Invitrogen; Thermo Fisher Scientific, Inc.) according to the manufacturer's protocol and negative control (cat. no. siN05815122147-1-5; Guangzhou RiboBio Co., Ltd.). Then U251 cells were incubated at $37^{\circ} \mathrm{C}$ in a $\mathrm{CO}_{2}$ incubator for $4 \mathrm{~h}$. Following this, the transfection mixture was replaced with fresh MEM medium. After 48-h transfection, U251 cells were used for further studies.

Western blot analysis. For western blot analysis, glioma tissues or cells were lysed for $30 \mathrm{~min}$ at $4^{\circ} \mathrm{C}$ using RIPA lysis buffer containing ethylenediaminetetraacetic acid-free protease inhibitor cocktail and phosphatase inhibitor cocktails 1 . The 
total proteins were quantified through BCA protein concentration assay kit (Beyotime Institute of Biotechnology), and $40 \mu \mathrm{g}$ protein per lane was resolved on $12 \%$ sodium dodecyl-sulfate polyacrylamide gel electrophoresis, and transferred to a PVDF membrane through wet-type transblotting apparatus (Bio-Rad Laboratories, Inc., Hercules, CA, USA). Then the PVDF membrane was blocked with $5 \%$ non-fat dry milk diluted in phosphate-buffered saline solution for $1 \mathrm{~h}$ at $4^{\circ} \mathrm{C}$, and incubated with primary antibodies directed against HDAC4 $(1: 2,000)$, p21 (1:2,000), p27 (1:2,000), CDK1 (1:2,000), vimentin $(1: 2,000)$, E-cadherin $(1: 2,000), \beta$-catenin $(1: 2,000)$ and GAPDH $(1: 1,000)$ at $4^{\circ} \mathrm{C}$ overnight. Subsequently, the PVDF membrane was washed three times with tris-buffered saline with $0.1 \%$ Tween-20 and incubated with the corresponding secondary antibody at a 1:5,000 dilution at room temperature for $2 \mathrm{~h}$. Following this, the PVDF membrane was visualized using BeyoECL moon. The results were analyzed through Quantity One software V4.62 (Bio-Rad Laboratories, Inc.) to obtain the optical density ratio of target protein to GAPDH.

Cell counting kit-8 (CCK-8) assay. A CCK-8 assay was used to assess the effects of HDAC4 on the proliferation of U251 cells. Briefly, $200 \mu 1 \mathrm{U} 251$ cells $\left(2 \times 10^{3}\right)$ were seeded in 96-well plates. At 0, 1, 2 and 4 days following transfection (overexpression or knockdown), the U251 cells were incubated with $20 \mu \mathrm{l}$ CCK- 8 solution at $37^{\circ} \mathrm{C}$ for $2 \mathrm{~h}$. The absorbance was examined at a wavelength of $450 \mathrm{~nm}$ on a 96 -well micro test spectrophotometer (BioTek Instruments, Inc., Winooski, VT, USA). The experiments were performed in triplicate.

Cell cycle analysis. The effects of HDAC4 on the cell cycle of U251 cells were assessed using flow cytometry (with the flow cytometer supplied by BD Biosciences, Franklin Lakes, NJ, USA). First, U251 cells were harvested using centrifugation for $10 \mathrm{~min}$ at $4^{\circ} \mathrm{C}$ and $1,200 \mathrm{x} \mathrm{g}$, synchronized by incubation at $4^{\circ} \mathrm{C}$ in serum-free MEM medium for $24 \mathrm{~h}$ and incubated with complete MEM medium for $24 \mathrm{~h}$ at $4^{\circ} \mathrm{C}$. Subsequently, U251 cells were washed twice with PBS and fixed with cold $70 \%$ ethanol at $-20^{\circ} \mathrm{C}$ overnight. U251 cells were washed twice with cold PBS and resuspended in PBS containing $100 \mu \mathrm{g} / \mathrm{ml}$ RNase $\mathrm{A}$ at $4^{\circ} \mathrm{C}$ for $30 \mathrm{~min}$. Then the resuspended $\mathrm{U} 251$ cells were further incubated with $100 \mu \mathrm{g} / \mathrm{ml}$ PI at $4^{\circ} \mathrm{C}$ for $30 \mathrm{~min}$. Finally, the cell cycle was analyzed through flow cytometry. The analysis was performed in triplicate with CELLQuestPro v5.1 (BD Biosciences).

ATP levels assay. Intracellular ATP levels were determined using an ATP assay kit according to the manufacturer's protocol. Briefly, U251 cells were lysed with ATP detection lysis buffer for $2 \mathrm{~min}$ at $4^{\circ} \mathrm{C}$ and centrifuged at $12,000 \mathrm{x} \mathrm{g}$ for $5 \mathrm{~min}$ at $4^{\circ} \mathrm{C}$. The supernatants were then mixed with the working solution of ATP detection reagent, and the relative fluorescence intensity was immediately assessed using a luminometer (Thermo Scientific Luminoskan Ascent; Thermo Fisher Scientific, Inc.). The fluorescence intensity was normalized by per sample protein content, which was measured through a BCA assay kit.

ROS measurement. Production of intracellular ROS was determined using a reactive oxygen species assay kit according to the manufacturer's protocol. Briefly, U251 cells were seeded in $60-\mathrm{mm}$ dishes and allowed to attach overnight. Then $10 \mathrm{mM}$ 2',7'-dichlorofluorescin diacetate (DCFH-DA) was added and incubated with the $\mathrm{U} 251$ cells for $20 \mathrm{~min}$ at $37^{\circ} \mathrm{C}$. Serum-free cell culture medium was used to wash the U251 cells three times to fully remove the inaccessible DCFH-DA. Once the U251 cells were collected, the fluorescence in U251 cells was assessed through a fluorescence spectrometer at $488 \mathrm{~nm}$ (excitation wavelengths) and $525 \mathrm{~nm}$ (emission wavelengths). ROS levels in U251 cells were expressed as relative intensity of DCF fluorescence per sample protein content.

Cell invasion assays. The invasive ability of U251 cells was assessed through 24 well Transwell permeable filters (Costar; Corning Incorporated) with $8 \mu \mathrm{M}$ pores. Briefly, the filter was washed with serum-free MEM medium, and then the upper side of the filter was evenly coated with $20 \mu$ l Matrigel (1:2 dilution with MEM). The chamber was divided into the upper chamber and the lower chamber. For invasion assays, subsequent to transfection with HDAC4 siRNA, pcDNA3.1-HDAC4 or negative control, U251 cells $\left(1 \times 10^{5}\right)$ were resuspended in serum free MEM medium and seeded into the upper chamber of the Transwell invasion system. The lower chamber was filled with complete medium. Following this, the Transwell invasion system was incubated for $48 \mathrm{~h}$ at $37^{\circ} \mathrm{C}$ in an incubator. Those U251 cells that invaded into the filter were detached from filter by trypsinization and that invaded in the lower chamber were collected and analyzed using an 3-(4,5-dimethylthiazol-2-yl)2,5-diphenyltetrazolium bromide (MTT) assay. Briefly, U251 cells were incubated with MTT $(5 \mathrm{mg} / \mathrm{ml})$ for $4 \mathrm{~h}$ at $37^{\circ} \mathrm{C}$. Then $150 \mu 1$ dimethyl sulfoxide was added to dissolve the crystals. The absorbance values were determined at $570 \mathrm{~nm}$ and $630 \mathrm{~nm}$ on a 96-well micro test spectrophotometer (BioTek Instruments, Inc.). The analysis was performed in triplicate.

Statistical analysis. All data were presented as the mean \pm standard error of the mean from at least three independent experiments. All data were analyzed using an unpaired Student's t-test or one-way analysis of variance followed by Dunnett's test using SPSS17.0 software (SPSS, Inc., Chicago, IL, USA). $\mathrm{P}<0.05$ was considered to indicate a statistically significant difference.

\section{Results}

HDAC4 displayed elevated expression in glioma tissues and cell lines. The expression of HDAC4 protein was evaluated in glioma by western blot analysis. First, HDAC4 expression was analyzed in 12 paired glioma tissues and adjacent nontumor tissues and revealed that the expression of HDAC4 was significantly upregulated in glioma tissues compared with glioma-adjacent normal tissues $(\mathrm{P}<0.01$; Fig. 1A). Additionally, three glioma cell lines (U251, U-87MG and LN-18) were analyzed. HDAC4 displayed higher expression in the three glioma cell lines compared with the non-cancerous human glial cell line SVG p12 ( $\mathrm{P}<0.01$; Fig. 1B). These data revealed that the expression profile of HDAC4 in glioma tissues coincided with that in glioma cell lines. In other words, the results of the present study demonstrated that the expression of HDAC4 was upregulated in glioma tissues and cell lines. 
A

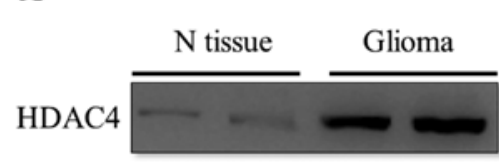

GAPDH

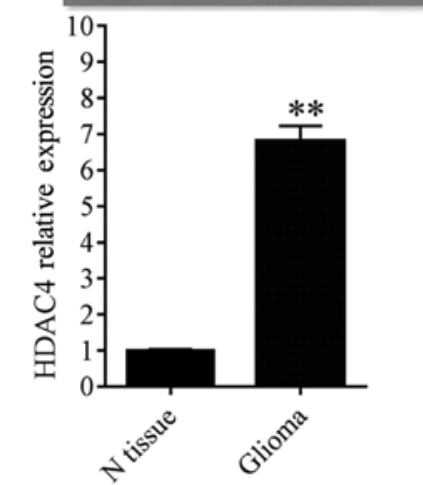

B
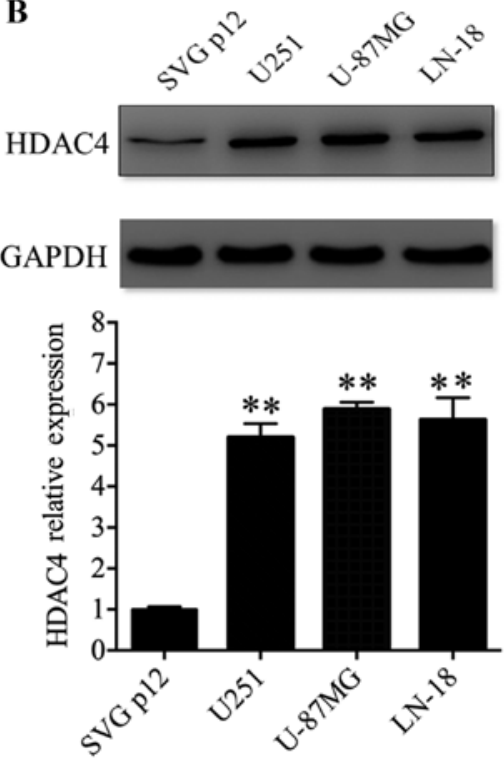

Figure 1. Expression of HDAC4 in glioma tissues and cell lines. Protein levels were determined using western blot analysis in glioma tissues and cell lines. (A) Significant upregulation of HDAC4 in glioma tissues compared with adjacent $\mathrm{N}$ tissues. ** $\mathrm{P}<0.01 \mathrm{vs}$. $\mathrm{N}$ tissue ( $\mathrm{n}=12$ ). (B) Elevated expression of HDAC4 in glioma cell lines (U251, U-87MG and LN-18). Data were presented as the mean \pm standard error of the mean. ${ }^{* *} \mathrm{P}<0.01 \mathrm{vs.} \mathrm{SVG} \mathrm{p12} \mathrm{cells.} \mathrm{HDAC4,} \mathrm{histone}$ deacetylase 4; N, non-tumor.

A
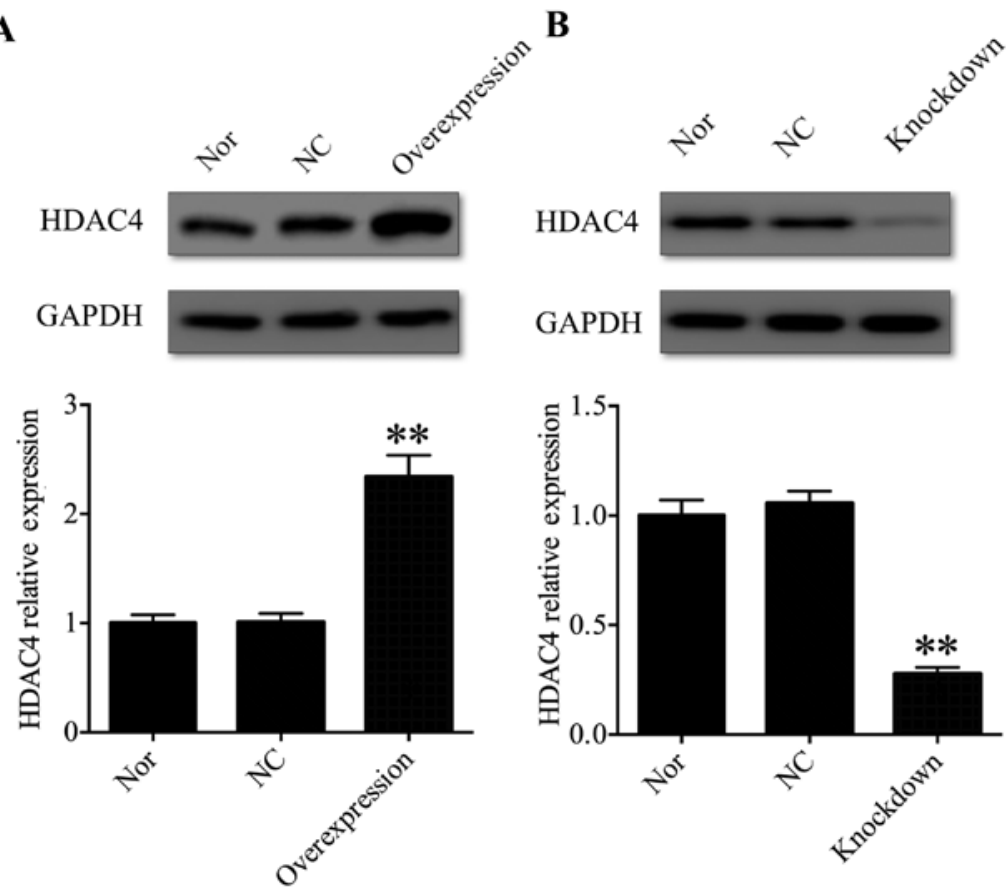

Figure 2. Expression of HDAC4 in transfected glioma U251 cells. The protein levels were determined through western blot analysis in glioma U251 cells transfected with pcDNA3.1-HDAC4 (overexpression) or HDAC4 small interfering RNA (knockdown). (A) Significantly higher expression of HDAC4 was observed in the overexpression group compared with normal U251 cells (Nor) or the U251 cells transfected with pcDNA3.1 vector (NC). ${ }^{* *} \mathrm{P}<0.01$ vs. Nor or NC. (B) HDAC4 displayed a significantly lower expression in the knockdown group compared with normal U251 cells (Nor) or the U251 cells transfected with negative control (NC). Data were presented as the mean \pm standard error of the mean. ${ }^{* *} \mathrm{P}<0.01$ vs. Nor or NC. HDAC4, histone deacetylase 4; Nor, normal; $\mathrm{NC}$, negative control.

HDAC4 was successfully knocked down or overexpressed in U251 cells. In order to address the function of HDAC4 in the tumorigenesis and progression of glioma, U251 cells were selected for further investigation. A pcDNA3.1-HDAC4 expression vector was used to overexpress HDAC4 in U251 cells, and HDAC4 siRNA was used to inhibit the expression of HDAC4 in U251 cells. Western blot analysis was used to evaluate HDAC4 overexpression and gene-silencing efficacy in U251 cells. The expression of HDAC4 was significantly upregulated in the pcDNA3.1-HDAC4-transfected U251 cells compared with that in the non-transfected U251 cells or negative control transfected U251 cells ( $\mathrm{P}<0.01$; Fig. 2A). 
A

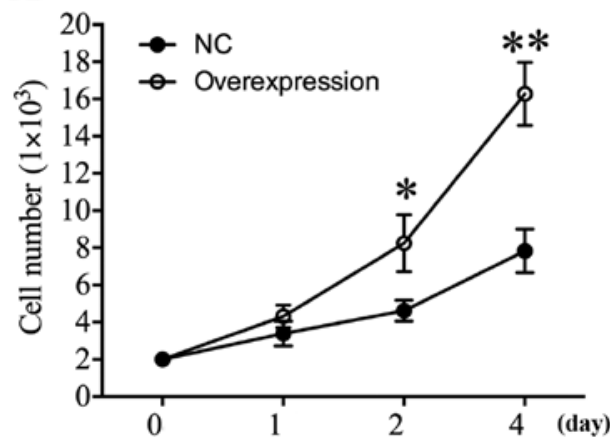

B

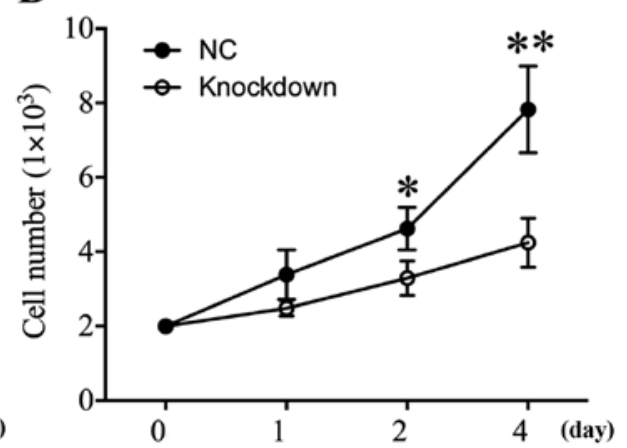

Figure 3. Effect of the HDAC4 on U251 cell proliferation. (A) Proliferation curve in U251 cells transfected with empty pcDNA3.1 vector (NC) or pcDNA3.1-HDAC4 (overexpression). (B) Proliferation curve in U251 cells transfected with HDAC4 siRNA (knockdown) or with siRNA negative control (NC). Data were expressed as the mean \pm standard error of the mean. ${ }^{*}<0.05$ and ${ }^{* *} \mathrm{P}<0.01 \mathrm{vs}$. NC. HDAC4, histone deacetylase 4; siRNA, small interfering RNA; $\mathrm{NC}$, negative control.

A

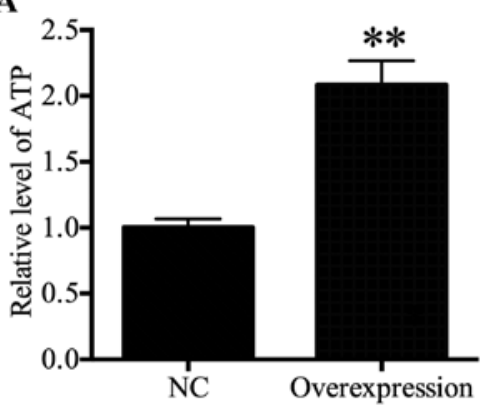

C

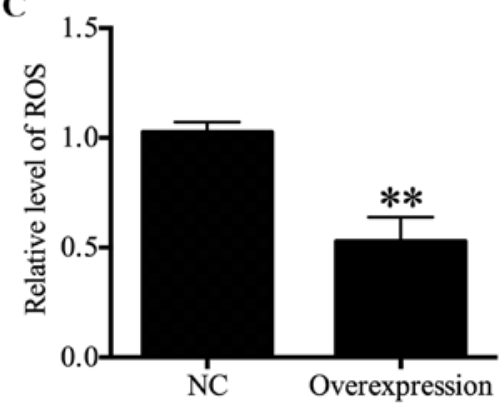

B

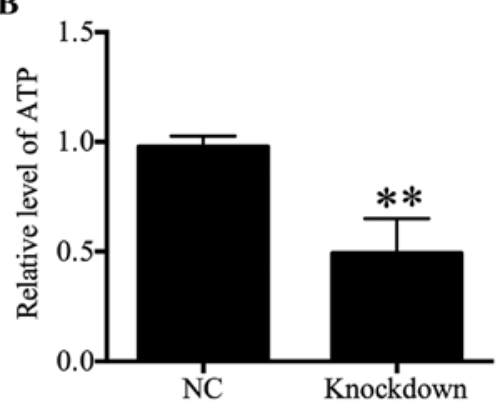

D

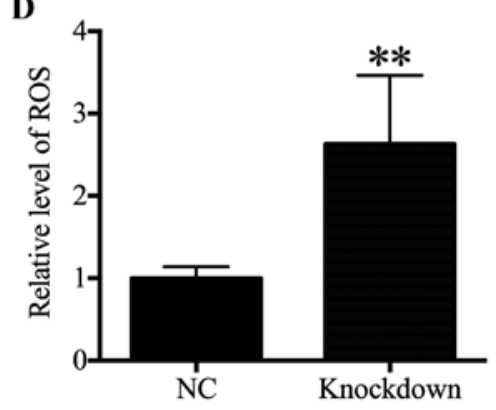

Figure 4. Effect of the HDAC4 on the generation of ATP and ROS in U251 cells. Generation of ATP in (A) HDAC4 overexpressing and (B) HDAC4 knockdown U251 cells compared with U251 cells transfected with empty pcDNA3.1 vector. Generation of ROS in (C) HDAC4 overexpressing and (D) HDAC4 knockdown U251 cells compared with U251 cells transfected with siRNA negative control. Overexpression indicates the U251 cells transfected with pcDNA3.1-HDAC4 and knockdown indicates the U251 cells transfected with HDAC4 siRNA. Data were expressed as the mean \pm standard error of the mean. *"P<0.01 vs. NC. HDAC4, histone deacetylase 4; ATP, adenosine triphosphate; ROS, reactive oxygen species; NC, negative control; siRNA, small interfering RNA.

Conversely, the expression of HDAC4 was significantly downregulated in the HDAC4 siRNA-transfected U251 cells compared with the non-transfected U251 cells or negative control transfected U251 cells $(\mathrm{P}<0.01$; Fig. 2B). These data suggested that HDAC4 was successfully knocked down or overexpressed in U251 cells.

HDAC4 promoted U251 cell proliferation. In order to investigate the effect of HDAC4 on the proliferation of glioma cells, CCK-8 analysis was performed at 24, 48 and $96 \mathrm{~h}$. The proliferation curves demonstrated that when HDAC4 was overexpressed in U251 cells, the cell proliferation was significantly increased 2.0-fold in comparison with U251 cells transfected with empty pcDNA3.1 vector on day $4(\mathrm{P}<0.01$; Fig. $3 \mathrm{~A})$.
Additionally, the effect of HDAC4 knockdown on cell proliferation in U251 cells was examined. The results revealed that HDAC4 knockdown significantly suppressed the proliferation capacity of U251 cells ( $\mathrm{P}<0.05$; Fig. 3B). In summary, these data suggested that elevated HDAC4 may serve a crucial role in the augmentation of the proliferation ability of U251 cells.

HDAC4 increased ATP levels and decreased ROS generation. Intracellular ATP levels were associated with proliferation (21-23). Therefore, ATP content was determined in the present study, and results revealed that a significant increase in the ATP content in HDAC4 overexpressing U251 cells was observed compared with the U251 cells transfected with empty pcDNA3.1 vector $(\mathrm{P}<0.01$; Fig. $4 \mathrm{~A})$. Furthermore, the 

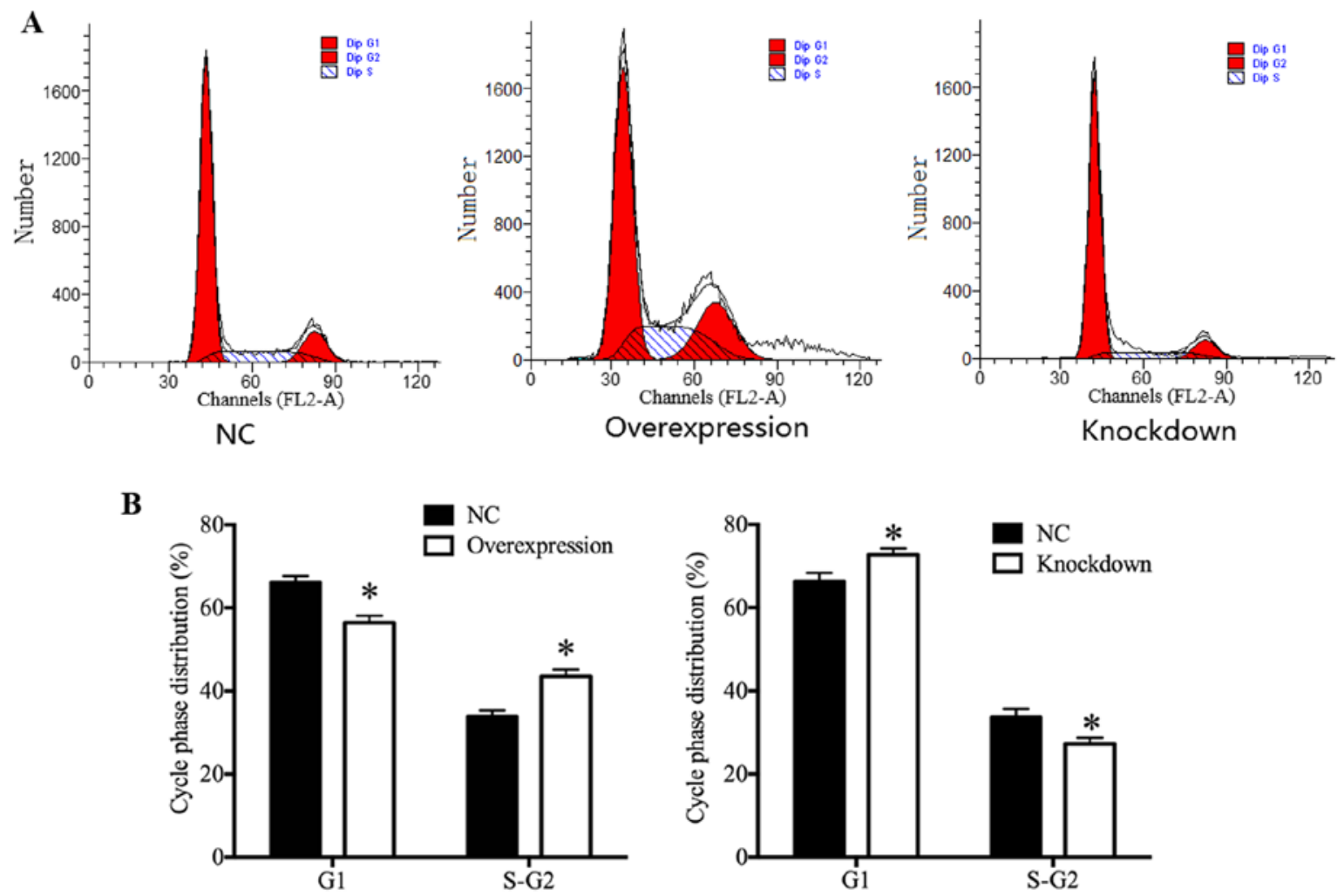

Figure 5. Effect of HDAC4 on the cell cycle distribution U251 cells. (A) Representative flow cytometry plots of the cell cycle. (B) Histogram (left) comparing the cell cycle distribution in the overexpression group and empty pcDNA3.1 vector group. Histogram (right) comparing the cell cycle distribution in the knockdown group and small interfering RNA NC group. The percentage of U251 cell cycle distribution was expressed as the mean \pm standard error of the mean. ${ }^{*} \mathrm{P}<0.05$ vs. NC. All experiments were performed in triplicate. HDAC4, histone deacetylase 4 ; NC, negative control.

ATP content reduced significantly in HDAC4 knockdown U251 cells $(\mathrm{P}<0.01 ;$ Fig. 4B. These data indicated that HDAC4 enhanced ATP generation. As intracellular ROS generation may be associated with mitochondrial dysfunction and ATP generation $(24,25)$, the effects of HDAC4 on ROS generation in U251 cells were further examined. The results indicated that ROS content displayed a significant decrease in the U251 cells transfected with pcDNA3.1-HDAC4 compared with the U251 cells transfected with empty pcDNA3.1 vector $(\mathrm{P}<0.01$; Fig. 4C). Meanwhile, downregulation of HDAC4 significantly activated ROS generation in U251 cells $(\mathrm{P}<0.01$; Fig. 4D). These results demonstrated that HDAC4 inhibited ROS generation in U251 cells.

HDAC4 promoted U251 cell cycle progression. In order to investigate whether the expression of HDAC4 was associated with the cell cycle progression of glioma U251 cells, the cell cycle distribution of glioma U251 cells was assessed by flow cytometry. Results demonstrated that HDAC4 overexpression exhibited a significant increase in the proportion of U251 cells at the $\mathrm{S}$ and $\mathrm{G} 2$ phase, and a significant decrease in the proportion of $\mathrm{U} 251$ cells at the G1 phase ( $\mathrm{P}<0.05$; Fig. 5A and B). On the contrary, there were significantly fewer U251 cells at the $\mathrm{S}$ and $\mathrm{G} 2$ phase, and significantly more $\mathrm{U} 251$ cells at the G1 phase in the HDAC4 knockdown group $(\mathrm{P}<0.05$; Fig. 5A and $\mathrm{B}$ ), suggesting that HDAC4 knockdown induced U251 cells $\mathrm{G} 1$ arrest. The cell cycle distribution results demonstrated that HDAC4 served a central role in the promotion of U251 cell cycle progression.
As p21, p27, CDK1 and CDK2 are associated with cell proliferation and cell cycle (26-28), the expression of p21, p27, CDK1 and CDK2 were determined in U251 cells. The present results revealed that cyclin-dependent kinase inhibitors p21 and p27 displayed a significant decrease in the HDAC4 overexpressing group, and a significant increase in HDAC4 knockdown group compared with their respective negative controls ( $\mathrm{P}<0.05$; Fig. 6). On the contrary, cyclin-dependent kinases CDK1 and CDK2 displayed an opposite expression pattern to $\mathrm{p} 21$ and $\mathrm{p} 27$, which demonstrated that the expression of CDK1 and CDK2 was significantly enhanced in the HDAC4 overexpressing group, and was significantly inhibited in the HDAC4 knockdown group $(\mathrm{P}<0.05$; Fig. 6$)$. These data indicated that HDAC4 enhanced the expression of cyclin-dependent kinases CDK1 and CDK2, and suppressed the expression of cyclin-dependent kinase inhibitors p21 and p27.

HDAC4 promoted the invasiveness of U251 cells. Subsequently, the function of HDAC4 in the invasiveness of U251 cells was determined through a Transwell invasion assay. The results suggested that the invasive ability of U251 cells was significantly enhanced in the HDAC4 overexpression group compared with in the empty pcDNA3.1 vector group $(\mathrm{P}<0.01$; Fig. 7A). On the contrary, the downregulation of HDAC4 exhibited a significant decrease in the invasive capacity of U251 cells compared with the siRNA negative control group $(\mathrm{P}<0.01$; Fig. 7B). These data suggested that HDAC4 strengthened the invasive ability of U251 cells. 
A

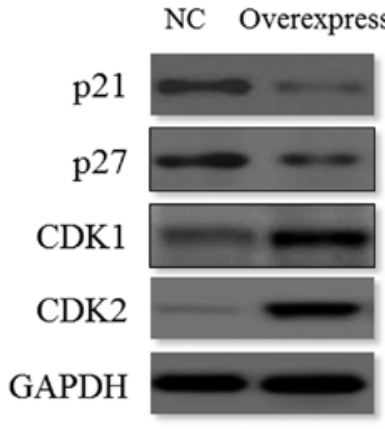

C

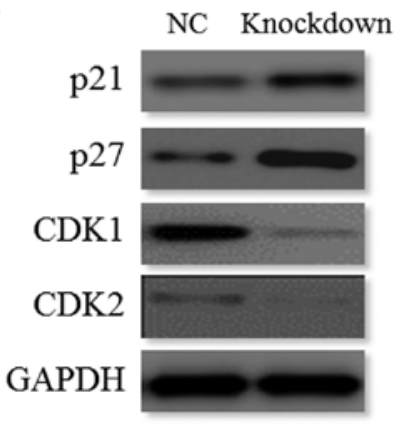

B

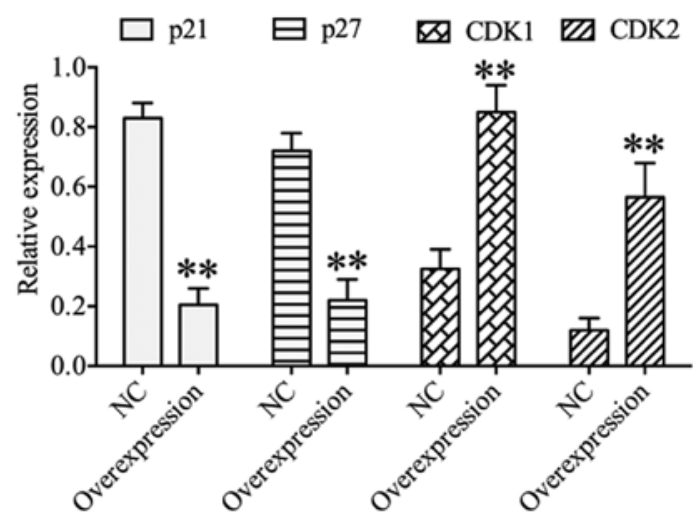

D

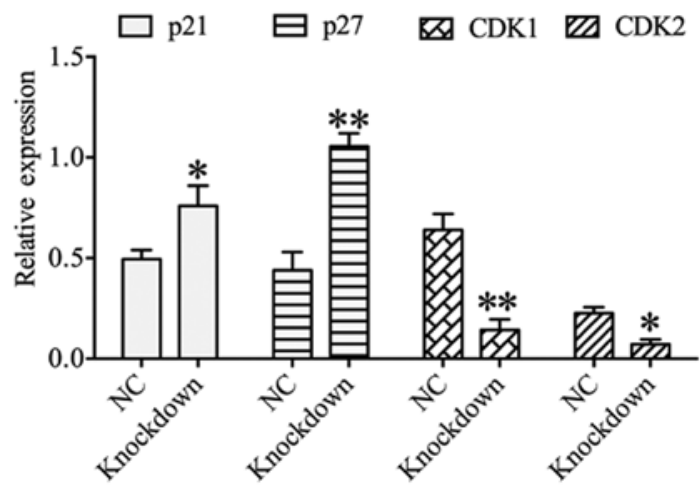

Figure 6. Effect of HDAC4 on the expression of p21, p27, CDK1 and CDK2 in U251 cells. (A) Expression of p21, p27, CDK1 and CDK2 in U251 cells transfected with pcDNA3.1-HDAC4 or empty pcDNA3.1 vector, and (B) quantified western blot analysis results. (C) Expression of p21, p27, CDK1 and CDK2 in U251 cells transfected with HDAC4 siRNA or siRNA negative control, and (D) quantified western blot analysis results. Overexpression indicates the U251 cells transfected with pcDNA3.1-HDAC4 and knockdown indicates the U251 cells transfected with HDAC4 siRNA. Data were expressed as the mean \pm standard error of the mean. ${ }^{*} \mathrm{P}<0.05$ and ${ }^{* *} \mathrm{P}<0.01$ vs. NC. HDAC4, histone deacetylase 4; NC, negative control; CDK1, cyclin-dependent kinase 1; CDK2, cyclin-dependent kinase 2; siRNA, small interfering RNA.

A

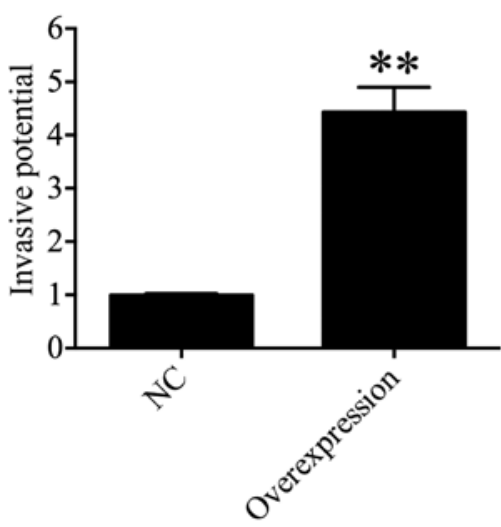

B

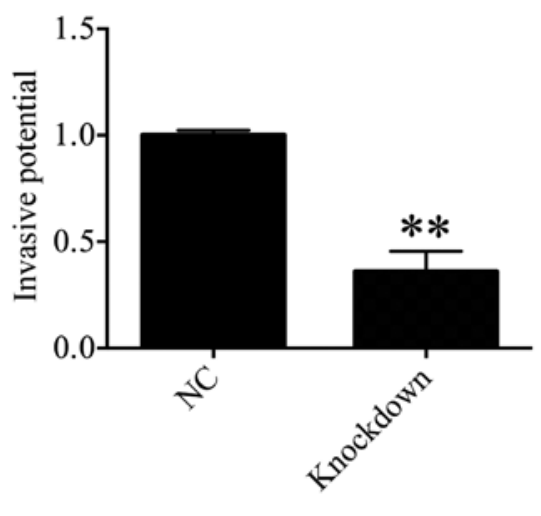

Figure 7. Effect of HDAC4 on U251 cell invasion. Histogram compared the invasive potential of U251 cells among different groups. (A) Invasive potential of U251 cells transfected with pcDNA3.1-HDAC4 or empty pcDNA3.1 vector. (B) Invasive potential of U251 cells transfected with HDAC4 siRNA or siRNA negative control. Data were expressed as the mean \pm standard error of the mean. ${ }^{* *} \mathrm{P}<0.01 \mathrm{vs.} \mathrm{NC.} \mathrm{All} \mathrm{experiments} \mathrm{were} \mathrm{performed} \mathrm{in} \mathrm{triplicate.} \mathrm{HDAC4,}$ histone deacetylase 4; NC, negative control; siRNA, small interfering RNA.

Altogether, these results indicated thee functional importance of HDAC4 in the invasiveness of U251 cells. Therefore, whether there exists any evidence for these molecular events in the invasiveness of U251 cells was questioned. Park et al (29) demonstrated that HDAC4 may activate the EMT in airway epithelial cells, and that HDAC4 knockdown may reduce EMT. Therefore, EMT markers, including vimentin, $\beta$-catenin and E-cadherin, were investigated and results indicated that 
A

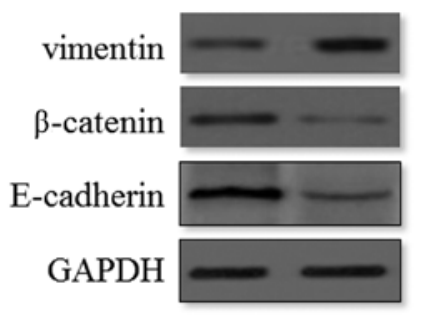

C

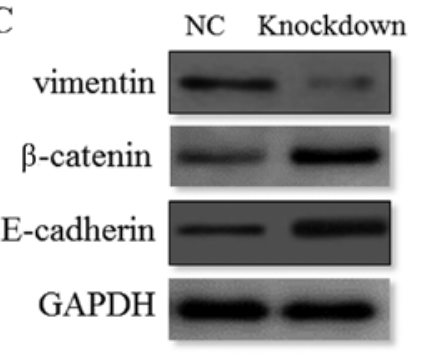

B

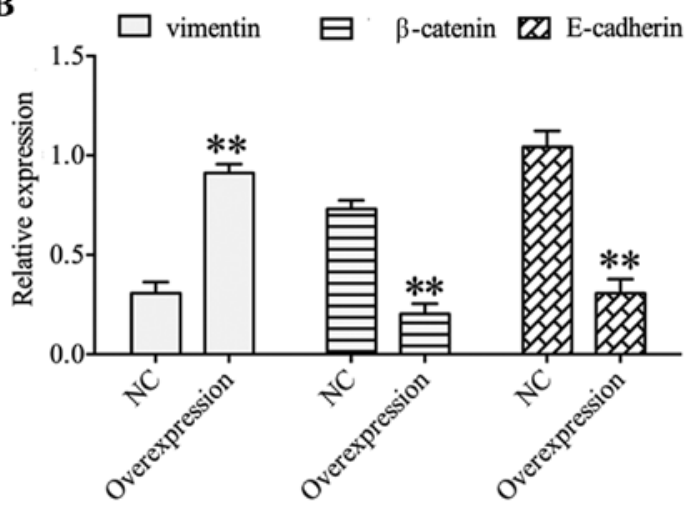

D

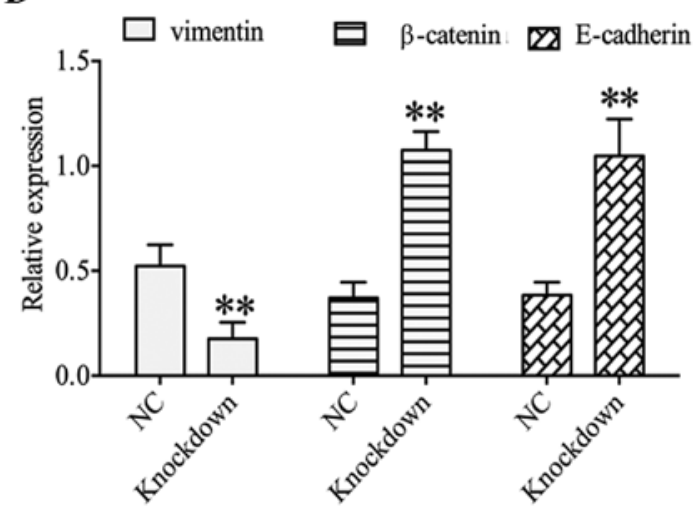

Figure 8. Effect of HDAC4 on the expression of vimentin, $\beta$-catenin and E-cadherin in U251 cells. (A) Expression of vimentin, $\beta$-catenin and E-cadherin in U251 cells transfected with pcDNA3.1-HDAC4 or empty pcDNA3.1 vector and (B) quantified western blot results. (C) Expression of vimentin, $\beta$-catenin and E-cadherin in U251 cells transfected with HDAC4 siRNA or siRNA negative control and (D) quantified western blot results. Data were expressed as the mean \pm standard error of the mean. ${ }^{*} \mathrm{P}<0.05$ and ${ }^{* * *} \mathrm{P}<0.01$ vs. NC. HDAC4, histone deacetylase 4; siRNA, small interfering RNA; NC, negative control.

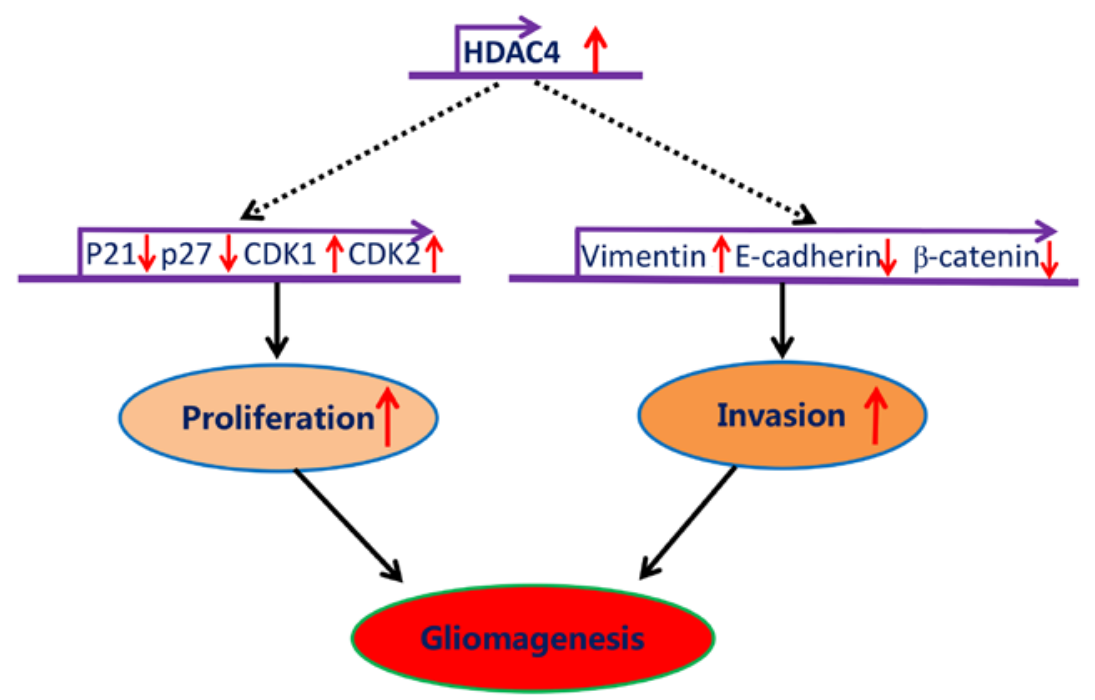

Figure 9. A model presenting the function of HDAC4 in the tumorigenesis of glioma. The integrated activation of CDK1, CDK21 and vimentin, and the suppression of $\mathrm{p} 21, \mathrm{p} 27, \beta$-catenin and E-cadherin by HDAC4 results in the tumorigenesis of glioma. HDAC4, histone deacetylase 4; CDK1, cyclin-dependent kinase 1; CDK2, cyclin-dependent kinase 2.

the expression of vimentin was significantly upregulated in the HDAC4 overexpression group and was significantly downregulated in the HDAC4 knockdown group compared with their respective negative controls $(\mathrm{P}<0.01$; Fig. 8). Nevertheless, the expression of E-cadherin and $\beta$-catenin was negatively associated with the expression of vimentin expression, which revealed that the expression of E-cadherin and $\beta$-catenin was significantly reduced in the HDAC4 overexpression group and was significantly enhanced in the HDAC4 knockdown group compared with the negative control $(\mathrm{P}<0.01$; Fig. 8). These data 
indicated that the aberrant expression of vimentin, $\beta$-catenin and E-cadherin were potential markers of the malignant transformation of glioma.

\section{Discussion}

Glioma is the most lethal type of primary malignant brain tumor. Although the current treatment modalities have progressed substantially, the recurrence of glioma remains a therapeutic challenge (30). Therefore, the survival of patients with glioma remains unsatisfactory. This is mainly due to the fact that the molecular mechanisms of glioma are unclear $(31,32)$. Growing evidence has revealed that HDAC4 was frequently dysregulated in human malignancies (12-15). HDAC5 are increased in human glioma tissues and promoted the proliferation of glioma cells by the upregulation of Notch 1 (33). However, the involvement of HDAC4 in gliomagenesis, migration and invasiveness remains elusive.

In the present study, the results revealed that the expression of HDAC4 was upregulated in glioma tissues and cell lines, which was consistent with the expression profile of HDAC4 in gastric cancer, colorectal cancer, lung cancer and ovarian cancer (14,34-37). These data indicated that elevated HDAC4 may serve an important role in gliomagenesis and progression. In order to address the function of HDAC4 in glioma cells, the glioma U251 cells were selected for further investigation. Furthermore, HDAC4 overexpression or knockdown was performed in U251 cells. Western blot analysis revealed that HDAC4 displayed a higher expression in the HDAC4 overexpression group and a lower expression in the HDAC4 knockdown group, which suggested that HDAC4 was successfully overexpressed or knockdown in U251 cells. Following this, the effects of HDAC4 on the cell proliferation, ATP level, ROS generation, cell cycle and invasion of U251 cells were determined. The results of the present study revealed that the HDAC4 may serve a crucial role in augmenting the proliferation ability of U251 cells and in the promotion of U251 cell cycle progression, which was consistent with and confirmed by previous studies from Zeng et al (17) and Kang et al $(17,34)$. Zeng et al revealed that HDAC4 was overexpressed in esophageal squamous cell carcinoma, and that HDAC4 overexpression was associated with advanced clinical stage and poor survival (17). Kang et al revealed that HDAC4 was upregulated in gastric cancer and that elevated HDAC4 promoted the proliferation of SGC-7901 cells, but HDAC4 knockdown inhibited the proliferation of SGC-7901 cells, resulting in cell cycle arrest at G0/G1 phase in SGC-7901 cells (34). As intracellular ATP levels were associated with cell proliferation and intracellular ROS generation was associated with mitochondrial dysfunction and served an important regulatory role in cell death and proliferation $(21-25,38)$, the present study therefore examined the effects of HDAC4 on intracellular ATP levels and ROS generation in U251 cells. The results of the present study indicated that HDAC4 enhanced ATP generation, but inhibited ROS generation in U251 cells, which was also consistent with and confirmed by a previous study on gastric cancer (34). Elevated HDAC4 in gastric cancer promoted the ATP levels in gastric cancer cells, but HDAC4 knockdown inhibited the ATP levels of gastric cancer cells, resulting in cell cycle arrest at the G0/G1 phase and ROS increase in gastric cancer cells (34). Thus, it was speculated that lower ATP levels and higher ROS levels may prevent the proliferation and tumorigenesis. The present data indicated that HDAC4 improved the cell proliferation and cycle progression of U251 cells through enhancing the ATP generation and suppressing the ROS generation.

Cyclin-dependent kinase inhibitors (p21 and p27) and cyclin-dependent kinases (CDK1 and CDK2) were associated with cell proliferation and the cell cycle (26-28). The present data indicated that HDAC4 enhanced the expression of cyclindependent kinases CDK1 and CDK2, and suppressed the expression of cyclin-dependent kinase inhibitors p21 and p27, which was also consistent with and confirmed by previous studies. Zeng et al (17) demonstrated that HDAC4 promoted the proliferation of esophageal carcinoma cell and G1/S cell cycle progression by inhibiting p21 and p27 and upregulating CDK2 and CDK4. Mottet et al (39) reported that HDAC4 participated in the repression of p21 in a human glioblastoma model. These data suggested that HDAC4 improved the cell proliferation and cycle progression of U251 cells through enhancing the expression of CDK1 and CDK2 and suppressing the expression of $\mathrm{p} 21$ and $\mathrm{p} 27$, and was accompanied by an increase in ATP levels and reduced ROS levels. However, the potential regulatory mechanism of HDAC4 in the expression of CDK1, CDK2, p21 and p27 also needs to be investigated further.

Glioma is characterized by diffuse infiltration $(1,2,4)$. Therefore, the present study determined the effects of HDAC4 on the invasiveness of U251 cells and the results revealed that HDAC4 strengthened the invasive ability of U251 cells. The results were confirmed by the study by Park et al (40), which demonstrated that the high expression of HDAC4 was associated with the high invasion of MDA-MB-231 cells. Ahn et al (41) revealed that the repression of MMP-2 was associated with the reduction of HDAC4 in ovarian cancer. Generally speaking, these data suggested the functional importance of HDAC4 in the invasiveness of U251 cells. One previous study demonstrated that HDAC4 may activate EMT in airway epithelial cells, and that HDAC4 knockdown may reduce EMT (29). Therefore, EMT markers, including vimentin, $\beta$-catenin and E-cadherin, were investigated and the results revealed that HDAC4 enhanced the expression of vimentin and suppressed the expression of E-cadherin and $\beta$-catenin in U251 cells. These data indicated that the aberrant expression of vimentin, $\beta$-catenin and E-cadherin were potential markers of the malignant transformation of glioma. However, the potential regulatory mechanism of HDAC4 in the expression of vimentin, $\beta$-catenin and E-cadherin also needs to be investigated further.

In addition, there are a number of shortcomings in the present study. First, the small sample size is a limitation of the study, and hence it is not possible to analyze the association between different grades and the expression of HDAC4 in glioma. Future studies will be conducted with a larger sample size to investigate this, which will substantiate the present study. Second, the expression of HDAC4 was detected only by western blot analysis; a morphological examination, for example immunohistochemistry, will be performed in future studies. Additionally, the present study only examined the protein expression of HDAC4 and relevant genes. In future 
studies, the mRNA level of the HDAC4 and relevant genes will be detected, which may further clarify whether abnormalities of these genes emerge in transcription and translation, or the translation only. All of this will render the present research conclusions more reliable.

In summary, the data generated by the present study demonstrated that HDAC4 was significantly upregulated in glioma, and promoted glioma cell proliferation and invasion mediated through, at least partially, the repression of p21, p27, E-cadherin and $\beta$-catenin, and by the potentiation of CDK1, CDK2 and vimentin (Fig. 9). Altogether, the present study revealed that HDAC4 overexpression was central for the tumorigenesis of glioma, which may serve as a useful prognostic biomarker and potential therapeutic target for glioma.

\section{Acknowledgements}

Not applicable.

\section{Funding}

The present study was supported by the Fund of Doctoral Start-up of Nantong University (grant no. 15B27) and the Nantong Science and Technology Department (key technology research project no. MS2201515110).

\section{Availability of data and materials}

All data generated or analyzed during this study are included in this published article.

\section{Authors' contributions}

JYC and XJN designed the study. JYC, TTX, YW, JJC, JL, $X Y C, X C$ and YFY performed the experiments. JJC, JL, XYC, $\mathrm{XC}$ and YFY collected and assembled the data. JYC and XJN performed the data analysis. XJN provided scientific expertise. JYC and XJN wrote the manuscript.

\section{Ethics approval and consent to participate}

This study was ethically approved by the Human Ethics Committee of The Affiliated Hospital of Nantong University (Nantong, China).

\section{Patient consent for publication}

All patients agreed to publication and provided written informed consent.

\section{Competing interests}

The authors declare that they have no competing interests.

\section{References}

1. Farshidfar Z, Faeghi F, Mohseni M, Seddighi A, Kharrazi HH and Abdolmohammadi J: Diffusion tensor tractography in the presurgical assessment of cerebral gliomas. Neuroradiol J 27: 75-84, 2014.
2. Kazakova MH, Staneva DN, Koev IG, Staikov DG, Mateva N, Timonov PT, Miloshev GA and Sarafian VS: Protein and mRNA levels of YKL-40 in high-grade glioma. Folia Biol (Praha) 60: 261-267, 2014.

3. Chen S, Han M, Chen W, He Y, Huang B, Zhao P, Huang Q, Gao L, Qu X and Li X: KIF1B promotes glioma migration and invasion via cell surface localization of MT1-MMP. Oncol Rep 35: 971-977, 2016.

4. Ortega-Aznar A, Jimenez-Leon P, MartinezE and Romero-Vidal FJ: Clinico-pathological and molecular aspects of diagnostic and prognostic value in gliomas. Rev Neurol 56: 161-170, 2013 (In Spanish)

5. Waitkus MS, Diplas BH and Yan H: Isocitrate dehydrogenase mutations in gliomas. Neuro-oncol 18: 16-26, 2016.

6. Lin M, Zhu Q, Wang J, Yang W, Fan H, Yi J and Jiang M: Molecules involved in acrosomal exocytosis and cortical granule exocytosis. Biotarget 1: 11, 2017.

7. Lindemann C, Hackmann O, Delic S, Schmidt N, Reifenberger G and Riemenschneider MJ: SOCS3 promoter methylation is mutually exclusive to EGFR amplification in gliomas and promotes glioma cell invasion through STAT3 and FAK activation. Acta Neuropathol 122: 241-251, 2011.

8. Fang Q, Xu T, Wu C, Zhou S and Sun H: Biotargets in neural regeneration. Biotarget 1: 6, 2017.

9. Crow M, Khovanov N, Kelleher JH, Sharma S, Grant AD, Bogdanov Y, Wood JN, McMahon SB and Denk F: HDAC4 is required for inflammation-associated thermal hypersensitivity. FASEB J 29: 3370-3378, 2015.

10. Cohen TJ, Choi MC, Kapur M, Lira VA, Yan Z and Yao TP: HDAC4 regulates muscle fiber type-specific gene expression programs. Mol Cells 38: 343-348, 2015.

11. Zhou J, Li P, Chen Q, Wei X, Zhao T, Wang Z and Wei L: Mitogen-activated protein kinase p38 induces HDAC4 degradation in hypertrophic chondrocytes. Biochim Biophys Acta 1853: $370-376,2015$

12. Xiao H, Jiao J, Wang L, O'Brien S, Newick K, Wang LC, Falkensammer E, Liu Y, Han R, Kapoor V, et al: HDAC5 controls the functions of Foxp3(+) T-regulatory and CD8(+) T cells. Int J Cancer 138: 2477-2486, 2016.

13. Hsieh TH, Hsu CY, Tsai CF, Long CY, Chai CY, Hou MF, Lee JN, Wu DC, Wang SC and Tsai EM: miR-125a-5p is a prognostic biomarker that targets HDAC4 to suppress breast tumorigenesis. Oncotarget 6: 494-509, 2015.

14. Wei JY, Li WM, Zhou LL, Lu QN and He W: Melatonin induces apoptosis of colorectal cancer cells through HDAC4 nuclear import mediated by CaMKII inactivation. J Pineal Res 58: 429-438, 2015.

15. Amodio N, Stamato MA, Gullà AM, Morelli E, Romeo E, Raimondi L, Pitari MR, Ferrandino I, Misso G, Caraglia M, et al: Therapeutic targeting of miR-29b/HDAC4 epigenetic loop in multiple myeloma. Mol Cancer Ther 15: 1364-1375, 2016.

16. Vallabhapurapu SD, Noothi SK, Pullum DA, Lawrie CH, Pallapati R, Potluri V, Kuntzen C, Khan S, Plas DR, Orlowski RZ, et al: Transcriptional repression by the HDAC4-RelB-p52 complex regulates multiple myeloma survival and growth. Nat Commun 6: 8428, 2015.

17. Zeng LS, Yang XZ, Wen YF, Mail SJ, Wang MH, Zhang MY, Zheng XF and Wang HY: Overexpressed HDAC4 is associated with poor survival and promotes tumor progression in esophageal carcinoma. Aging (Albany NY) 8: 1236-1249, 2016.

18. Kaowinn S, Jun SW, Kim CS, Shin DM, Hwang YH, Kim K, Shin B, Kaewpiboon C, Jeong HH, Koh SS, et al: Increased EGFR expression induced by a novel oncogene, CUG2, confers resistance to doxorubicin through Stat1-HDAC4 signaling. Cell Oncol (Dordr) 40: 549-561, 2017.

19. Louis DN, Ohgaki H, Wiestler OD, Cavenee WK, Burger PC, Jouvet A, Scheithauer BW and Kleihues P: The 2007 WHO classification of tumours of the central nervous system. Acta Neuropathol 114: 97-109, 2007.

20. Henriksen S, Tylden GD, Dumoulin A, Sharma BN, Hirsch HH and Rinaldo $\mathrm{CH}$ : The human fetal glial cell line SVG p12 contains infectious BK polyomavirus. J Virol 88: 7556-7568, 2014.

21. George J, Gonçalves FQ, Cristóvão G, Rodrigues L, Meyer Fernandes JR, Gonçalves T, Cunha RA and Gomes CA: Different danger signals differently impact on microglial proliferation through alterations of ATP release and extracellular metabolism. Glia 63: 1636-1645, 2015.

22. Kühn F: New insights into the interaction between ADP-ribose and human TRPM2 channel. Biotarget 1: 14, 2017. 
23. Almontashiri NA, Chen HH, Mailloux RJ, Tatsuta T, Teng AC, Mahmoud AB, Ho T, Stewart NA, Rippstein P, Harper ME, et al; CARDIoGRAM Consortium: SPG7 variant escapes phosphorylation-regulated processing by AFG3L2, elevates mitochondrial ROS, and is associated with multiple clinical phenotypes. Cell Reports 7: 834-847, 2014.

24. Jiang K, Wang W, Jin X, Wang Z, Ji Z and Meng G: Silibinin, a natural flavonoid, induces autophagy via ROS-dependent mitochondrial dysfunction and loss of ATP involving BNIP3 in human MCF7 breast cancer cells. Oncol Rep 33: 2711-2718, 2015.

25. Brookes PS, Yoon Y, Robotham JL, Anders MW and Sheu SS: Calcium, ATP, and ROS: A mitochondrial love-hate triangle. Am J Physiol Cell Physiol 287: C817-C833, 2004.

26. Van de Wouwer M, Couzinié C, Serrano-Palero M, GonzálezFernández O, Galmés-Varela C, Menéndez-Antolí P, Grau L and Villalobo A: Activation of the BRCA1/Chk1/p53/p21(Cip1/ Waf1) pathway by nitric oxide and cell cycle arrest in human neuroblastoma NB69 cells. Nitric Oxide 26: 182-191, 2012.

27. Wang J, Wang G and Khan MF: Disorder of G2-M Checkpoint control in aniline-induced cell proliferation in rat spleen. PLoS One 10: e0131457, 2015.

28. Yi X, Li Y,Zai H, Long X and Li W: KLF8 knockdown triggered growth inhibition and induced cell phase arrest in human pancreatic cancer cells. Gene 585: 22-27, 2016.

29. Park IH, Kang JH, Shin JM and Lee HM: Trichostatin A inhibits epithelial mesenchymal transition induced by TGF- $\beta 1$ in airway epithelium. PLoS One 11: e0162058, 2016.

30. Tu Y, Gao X, Li G, Fu H, Cui D, Liu H, Jin W and Zhang Y: MicroRNA-218 inhibits glioma invasion, migration, proliferation, and cancer stem-like cell self-renewal by targeting the polycomb group gene Bmil. Cancer Res 73: 6046-6055, 2013.

31. Le Rhun E, Taillibert S and Chamberlain MC: Anaplastic glioma: Current treatment and management. Expert Rev Neurother 15: 601-620, 2015.

32. Wirsching HG, Happold C, Roth P and Weller M: Management of diffusely infiltrating glioma in the elderly. Curr Opin Oncol 27: 502-509, 2015
33. Liu Q, Zheng JM, Chen JK, Yan XL, Chen HM, Nong WX and Huang HQ: Histone deacetylase 5 promotes the proliferation of glioma cells by upregulation of Notch 1. Mol Med Rep 10: 20452050, 2014

34. Kang ZH, Wang CY, Zhang WL, Zhang JT, Yuan CH, Zhao PW, Lin YY, Hong S, Li CY and Wang L: Histone deacetylase HDAC4 promotes gastric cancer SGC-7901 cells progression via p21 repression. PLoS One 9: e98894, 2014.

35. Kaewpiboon C, Srisuttee R, Malilas W, Moon J, Oh S, Jeong HG, Johnston RN, Assavalapsakul W and Chung YH: Upregulation of Stat1-HDAC4 confers resistance to etoposide through enhanced multidrug resistance 1 expression in human A549 lung cancer cells. Mol Med Rep 11: 2315-2321, 2015.

36. Isaacs JT, Antony L, Dalrymple SL, Brennen WN, Gerber S, Hammers H, Wissing M, Kachhap S, Luo J, Xing L, et al: Tasquinimod Is an aAllosteric modulator of HDAC4 survival signaling within the compromised cancer microenvironment. Cancer Res 73: 1386-1399, 2013.

37. Stronach EA, Alfraidi A, Rama N, Datler C, Studd JB, Agarwal R, Guney TG, Gourley C, Hennessy BT, Mills GB, et al: HDAC4regulated STAT1 activation mediates platinum resistance in ovarian cancer. Cancer Res 71: 4412-4422, 2011.

38. Chen EI: Mitochondrial dysfunction and cancer metastasis. J Bioenerg Biomembr 44: 619-622, 2012.

39. Mottet D, Pirotte S, Lamour V, Hagedorn M, Javerzat S, Bikfalvi A, Bellahcène A, Verdin E and Castronovo V: HDAC4 represses p21(WAF1/Cip1) expression in human cancer cells through a Sp1-dependent, p53-independent mechanism. Oncogene 28: 243-256, 2009.

40. Park SY, Jun JA, Jeong KJ, Heo HJ, Sohn JS, Lee HY, Park CG and Kang J: Histone deacetylases 1, 6 and 8 are critical for invasion in breast cancer. Oncol Rep 25: 1677-1681, 2011.

41. Ahn MY, Kang DO, Na YJ, Yoon S, Choi WS, Kang KW, Chung HY, Jung JH, Min S and Kim HS: Histone deacetylase inhibitor, apicidin, inhibits human ovarian cancer cell migration via class II histone deacetylase 4 silencing. Cancer Lett 325: 189-199, 2012. 